\title{
Fractal butterflies in buckled graphene-like materials
}

\author{
Vadym M. Apalkov \\ Department of Physics and Astronomy, Georgia State University, Atlanta, Georgia 30303, USA \\ Tapash Chakraborty \\ Department of Physics and Astronomy, University of Manitoba, Winnipeg, Canada R3T 2N2
}

(Dated: October 26, 2018)

\begin{abstract}
We study theoretically the properties of buckled graphene-like materials, such as silicene and germanene, in a strong perpendicular magnetic field and a periodic potential. We analyze how the spin-orbit interaction and the perpendicular electric field influences the energy spectra of these systems. When the magnetic flux through a unit cell of the periodic potential measured in magnetic flux quantum is a rational number, $\alpha=p / q$, then in each Landau level the energy spectra have a band structure, which is characterized by the corresponding gaps. We study the dependence of those gaps on the parameters of the buckled graphene-like materials. Although some gaps have weak dependence on the magnitude of the spin-orbit coupling and the external electric field, there are gaps that show strong nonomonotic dependence on these parameters. For $\alpha=1 / 2$, the spin-orbit interaction also opens up a gap at one of the Landau levels. The magnitude of the gap increases with spin-orbit coupling and decreases with the applied electric field.
\end{abstract}

\section{INTRODUCTION}

Electron dynamics in a periodic potential subjected to a perpendicular magnetic field was the subject of intensive theoretical and experimental research for several decades [2 29]. That interest on this particular subject derives from its unique energy spectrum, which as a function of the magnetic flux per lattice cell of the periodic potential reveals a fractal pattern, known as the Hofstadter's butterfly (due to the pattern resembling the butterflies). This is the first example of fractal pattern realized in a quantum system. The parameter which determines the fractal structure of the energy spectrum is the magnetic flux $\tilde{\alpha}$ through a unit cell measured in units of the magnetic flux quantum. In the case of a weak magnetic field $B$, first the periodic potential forms the Bloch bands and then the external magnetic field splits each Bloch band of the periodic potential into minibands of Landau level (LL) type, the number of which is determined by the parameter $\tilde{\alpha}$. In the strong magnetic field regime, the relevant parameter is $\alpha=1 / \tilde{\alpha}$ which is zero when $B \rightarrow \infty$. In this case, the energy spectra of an electron can be described by the formation of the LL spectrum and then splitting of the LL states into minibands by the periodic potential. Here the number of minibands is determined by the parameter $\alpha$, i.e., for rational $\alpha=p / q$ where $p$ and $q$ are integers, within a single LL there are $q$ minibands with $p$ degeneracy. To observe the fractal pattern in a reasonable range of the magnetic field, the important requirement is that the lattice structure has a large period. This can be achieved in artificial superlattices based on semiconductor nanostructures [6]. Observation of a clear fractal pattern remained a challenge however. Finally, in 2013 the Hofstadter butterfly structure with clear fractal pattern was reported [10 12] in monolayer and bilayer graphene [13]. In these experiments, the periodic lattice with a period $\approx 10 \mathrm{~nm}$ was created naturally by the moire pattern that appears when graphene is placed on hexagonal boron nitride with a twist [17, 18]. In addition to the natural formation of the period structure in graphene on boron nitride substrate, that system has also the unique relativistic energy dispersion, which results in special type of Landau levels [14 16].

Recently discovered new Dirac-type materials, such as silicene and germanene [19 27] bring in additional remarkable features and additional control parameters into the structure of the Dirac energy spectrum. These materials are monolayers of silicon and germanium with hexagonal lattice structures where the low energy charge carriers are also massless Dirac fermions 28 34 just as in graphene [14, 15]. Experimentally, the two-dimensional (2D) silicene was synthesized on $\mathrm{Ag}(111)$ [30 33] and zirconium diboride substrates [34], while germanene was grown on $\mathrm{Ag}$ [35, 36] and $\mathrm{Pt}$ [36] substrates. The main difference between silicene/germanene and graphene is that due to the larger radius of the $\mathrm{Si} / \mathrm{Ge}$ atom compared to the $\mathrm{C}$ atom, the corresponding hexagon lattices in germanene and silicene have buckled structure [37], i.e., the two sublattices (say $A$ and $B$ ) in these systems are displaced vertically by a finite distance $L_{z}$. As a result, silicene and germanene have large spin-orbit interactions, which opens the band gaps at the Dirac points $\left(\Delta_{\text {so }} \approx 1.55-7.9 \mathrm{meV}\right.$ for silicene [24, 38] and $\Delta_{\mathrm{so}} \approx 24-93 \mathrm{meV}$ for germanene [24, 38]). In the case of graphene, in contrast, the corresponding spin-orbitinduced gap is tiny, $25 \mu \mathrm{eV}$ [39]. The buckled structure of silicene/germanene lattice also allows for the band gap to be controlled by an applied perpendicular electric field [40] and the size of the band gap increases almost linearly with the electric field. These properties have important implications for a highly correlated electron systems in these graphene-like but novel systems [41], in particular, in the fractional quantum Hall effect regime 16, 42]. The buckled graphene-like materials with a strong spin-orbit interaction and sensitivity to the external electric field 


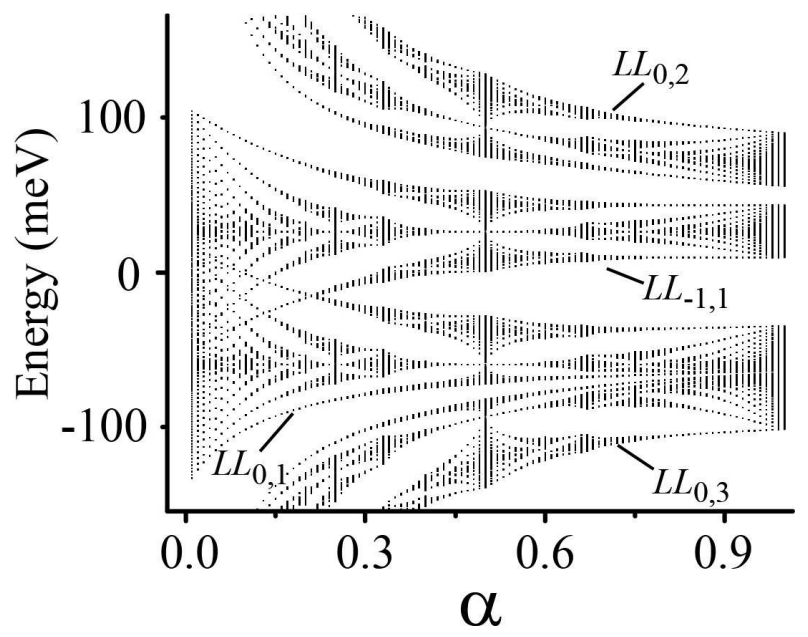

FIG. 1: Energy spectrum (Hofstadter butterfly) of germanene monolayer as a function of the parameter $\alpha$, which is the inverse magnetic flux through the unit cell in units of the flux quantum. The external electric field is $E_{z}=50 \mathrm{mV} / \AA$. The amplitude of the periodic potential is $V_{0}=40 \mathrm{meV}$ and its period is $a=20 \mathrm{~nm}$.

significantly modify the energy spectrum of the system, which should be also visible in the Hofstadter's butterfly pattern of these materials. Below we study the properties of the buckled graphene-like materials, silicene and germanene, placed in a perpendicular magnetic field and a periodic potential.

\section{THEORETICAL FRAMEWORK}

We consider an electron in a silicene/germanene monolayer in an external perpendicular magnetic field and a periodic potential. The Hamiltonian, $\mathcal{H}$, of such an electron consists of the kinetic energy term $\mathcal{H}_{0}$ and the periodic potential $V(x, y)$,

$$
\mathcal{H}=\mathcal{H}_{0}+V(x, y)
$$

In a magnetic field $B$, the kinetic energy part of the Hamiltonian has the following matrix form [43]

$\mathcal{H}_{0}=\left(\begin{array}{cccc}\Delta_{+}\left(E_{z}\right) & \hbar \omega_{c} \hat{a} & i \frac{\sqrt{2} \hbar a \lambda_{\mathrm{R}}}{\ell_{0}} \hat{a}^{+} & 0 \\ \hbar \omega_{c} \hat{a}^{+} & -\Delta_{+}\left(E_{z}\right) & 0 & -i \frac{\sqrt{2} \hbar a \lambda_{\mathrm{R}}}{\ell_{0}} \hat{a}^{+} \\ -i \frac{\sqrt{2} \hbar a \lambda_{\mathrm{R}}}{\ell_{0}} \hat{a} & 0 & \Delta_{-}\left(E_{z}\right) & \hbar \omega_{c} \hat{a} \\ 0 & i \frac{\sqrt{2} \hbar a \lambda_{\mathrm{R}}}{\ell_{0}} \hat{a} & \hbar \omega_{c} \hat{a}^{+} & -\Delta_{-}\left(E_{z}\right)\end{array}\right)$,

where $\lambda_{\mathrm{R}}$ is the Rashba spin-orbit constant, $\ell_{0}=$ $\sqrt{\hbar c / e B}$ is the magnetic length, $\omega_{c}=\sqrt{2} \hbar v_{\mathrm{F}} / \ell_{0}, v_{\mathrm{F}}$ is the Fermi velocity, $\Delta_{ \pm}=\mp \lambda_{\mathrm{SO}}+L_{z} E_{z}$. Here $\lambda_{\mathrm{SO}}$ is the spin-orbit constant, $2 L_{z}$ is the separation of two sublattices $A$ and $B$ in the $z$ direction, and $E_{z}$ is the external perpendicular electric field. For germanene and

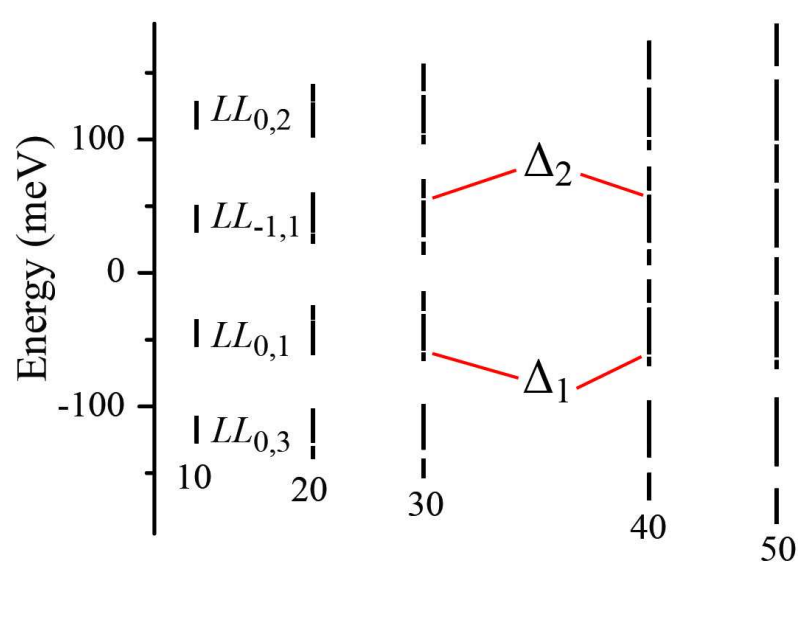

FIG. 2: Energy spectra of germanene monolayer for $\alpha=1 / 3$. The external electric field is $E_{z}=10 \mathrm{mV} / \AA$. The numbers near the lines are the amplitudes of the periodic potential, $V_{0}$. The period of the potential is $a=20 \mathrm{~nm}$. Two gaps $\Delta_{1}$ and $\Delta_{2}$ are marked in the figure.

silicene, the parameters in the above Hamiltonian are $v_{\mathrm{F}}=7.26 \times 10^{5} \mathrm{~m} / \mathrm{s}, L_{z}=0.33 \AA, \lambda_{\mathrm{SO}}=43 \mathrm{meV}$, $\lambda_{\mathrm{R}}=10.7 \mathrm{meV}$ for germanene and $v_{\mathrm{F}}=8.47 \times 10^{5} \mathrm{~m} / \mathrm{s}$, $L_{z}=0.23 \AA, \lambda_{\mathrm{SO}}=3.9 \mathrm{meV}, \lambda_{\mathrm{R}}=0.7 \mathrm{meV}$ for silicene.

The wavefunctions of the Hamiltonian (2) have four components and can be expressed in terms of the nonrelativistic wave functions $\phi_{n, k}$, which correspond to $n$-th conventional nonrelativistic Landau level and have the in-plane $y$ component of the wave vector $k$,

$$
|n k\rangle=\left(C_{1} \phi_{n, k}, C_{2} \phi_{n+1, k}, C_{3} \phi_{n-1, k}, C_{4} \phi_{n, k}\right) .
$$

In this basis the Hamiltonian (2) takes the form

$$
\mathcal{H}_{0}^{(n)}=\left(\begin{array}{cccc}
\Delta_{+}\left(E_{z}\right) & \kappa_{n+1} & i \alpha_{n} & 0 \\
\kappa_{n+1} & -\Delta_{+}\left(E_{z}\right) & 0 & -i \alpha_{n+1} \\
-i \alpha_{n} & 0 & \Delta_{-}\left(E_{z}\right) & \kappa_{n} \\
0 & i \alpha_{n+1} & \kappa_{n} & -\Delta_{-}\left(E_{z}\right)
\end{array}\right)
$$

where $\kappa_{n}=\hbar \omega_{c} \sqrt{n}, \alpha_{n}=\sqrt{2 n} \hbar a \lambda_{\mathrm{R}} / \ell_{0}$ and the wave functions are determined now by the coefficients $\left(C_{1}, C_{2}, C_{3}, C_{4}\right)$. form

The conventional Landau wave functions $\phi_{n, k}$ have the

$$
\phi_{n, k}(x, y)=\frac{e^{i k y}}{\sqrt{L}} \frac{e^{-\left(x-x_{k}\right)^{2} / 2 \ell_{0}^{2}}}{\sqrt{\pi^{1 / 2} \ell_{0} 2^{n} n !}} H_{n}\left(x-x_{k}\right),
$$

where $L$ is the length of a sample in the $y$ direction, $k$ is the $y$ component of the electron wave vector, $x_{k}=k \ell_{0}^{2}$, and $H_{n}(x)$ are the Hermite polynomials.

Depending on the value of $n$, the energy spectrum of the Hamiltonian (4) has the following properties: (i) for $n=-1$ there is only one Landau level, which is characterized by the wavefunction

$$
|-1, k\rangle=\left(0, \phi_{0, k}, 0,0\right)
$$




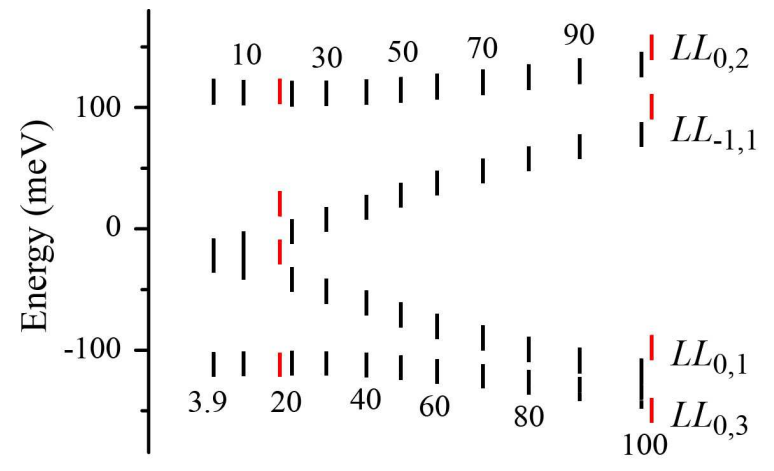

FIG. 3: Energy spectra of buckled graphene-like materials for $\alpha=1 / 3$. The external electric field is $E_{z}=100 \mathrm{mV} / \AA$ (black lines) and $E_{z}=0$ (red lines). The numbers near the lines are the SO interaction constants, $\lambda_{\mathrm{SO}}$. The period of the potential is $a=20 \mathrm{~nm}$ and its amplitude is $V_{0}=20 \mathrm{meV}$.

and has the energy of $E_{n=-1}=-\Delta_{+}\left(E_{z}\right)$; (ii) for $n=0$ there are three different Landau levels,

$$
|0, k\rangle=\left(C_{1} \phi_{0, k}, C_{2} \phi_{1, k}, 0, C_{4} \phi_{0, k}\right)
$$

and their energies are determined by the following cubic equation

$$
\begin{aligned}
& E^{3}+\Delta_{-} E^{2}-\left(\Delta_{+}^{2}+\lambda_{1}^{2}+\kappa_{1}^{2}\right) E \\
& +\left(\lambda_{1}^{2} \Delta_{+}-\Delta_{+}^{2} \Delta_{-}-\kappa_{1}^{2} \Delta_{-}\right)=0
\end{aligned}
$$

In the absence of the external electric field $\left(E_{z}=0\right)$, one of the solutions of Eq. (8) is $E_{0}=\Delta_{+}=-\lambda_{\mathrm{SO}}$. The corresponding wavefunction is

$$
|0, k\rangle=\left(i \alpha_{1} \phi_{0, k}, 0,0, \kappa_{1} \phi_{0), k}\right)
$$

and (iii) for $n>0$ there are four different Landau levels with the general structure $\left(C_{1} \phi_{n, k}, C_{2} \phi_{n+1, k}, C_{3} \phi_{n-1, k}, C_{4} \phi_{n, k}\right)$. In what follows, we mainly consider the properties of the $n=-1$ and $n=0$ Landau levels.

The periodic potential, $V(x, y)$, in the Hamiltonian (10) is characterized by its period $a_{0}$ and amplitude $V_{0}$. We assume that the potential has the following profile

$$
V(x, y)=V_{0}\left[\cos \left(q_{x} x\right)+\cos \left(q_{y} y\right)\right]
$$

where $q_{x}=q_{y}=q_{0}=2 \pi / a_{0}$. The periodic potential mixes the electron states $\Psi_{n, k}$ within a single LL, i.e., states with the same value of the LL index $n$ and different values of $k$, and also mixes the states of different LLs. The strength of this mixing is determined by the matrix elements of the periodic potential $V(x, y)$ between the LL states.

The matrix elements of the periodic potential in the basis of the LL wave functions of the buckled materials
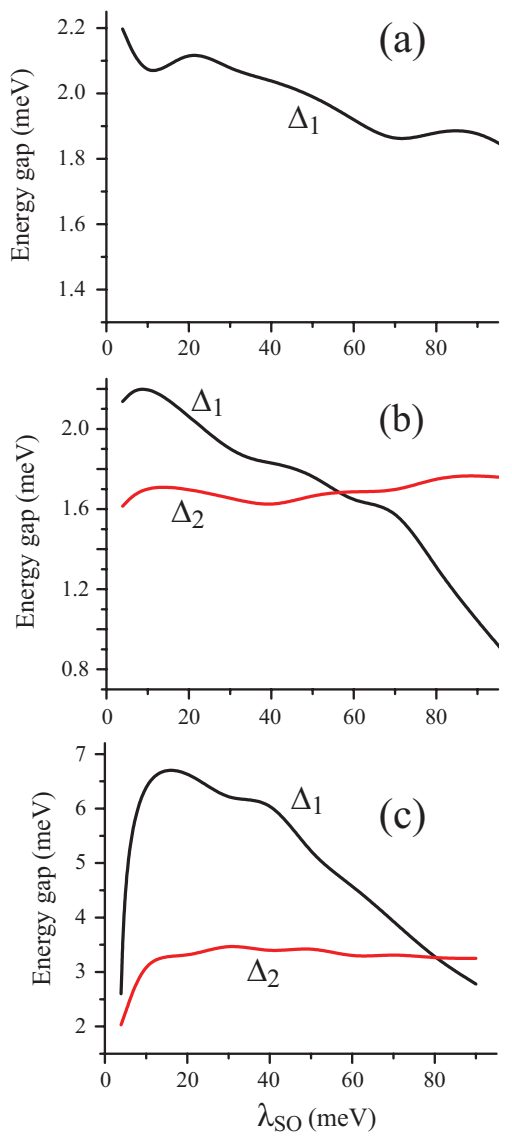

FIG. 4: Gaps $\Delta_{1}$ and $\Delta_{2}$, defined in Fig. 2] as functions of the SO interaction, $\lambda_{\mathrm{SO}}$. The period of the potential is $20 \mathrm{~nm}$. (a) The electric field is zero and the amplitude of periodic potential is $10 \mathrm{meV}$. (b) The electric field is $100 \mathrm{mV} / \AA$ and the amplitude of the periodic potential is $10 \mathrm{meV}$. (c) The electric field is $100 \mathrm{mV} / \AA$ and the amplitude of the periodic potential is $20 \mathrm{meV}$.

have the following form

$$
\begin{aligned}
& \left\langle n^{\prime} k^{\prime}\left|\cos \left(q_{0} y\right)\right| n k\right\rangle= \\
& \frac{i^{n-n^{\prime}}}{2}\left\{\delta_{k^{\prime}, k+q_{0}}+(-1)^{n-n^{\prime}} \delta_{k^{\prime}, k-q_{0}}\right\} \\
& \times\left[C_{n, 1} C_{n^{\prime}, 1} M_{\left|n^{\prime}\right|-1,|n|-1}+C_{n, 4} C_{n^{\prime}, 4} M_{\left|n^{\prime}\right|+1,|n|+1}\right. \\
& \left.+\left(C_{n, 2} C_{n^{\prime}, 2}+C_{n, 3} C_{n^{\prime}, 3}\right) M_{\left|n^{\prime}\right|,|n|}\right]
\end{aligned}
$$

and

$$
\begin{aligned}
& \left\langle n^{\prime} k^{\prime}\left|\cos \left(q_{0} x\right)\right| n k\right\rangle=\frac{\delta_{k^{\prime}, k}}{2}\left[e^{i q_{0} k \ell_{0}^{2}}+(-1)^{n-n^{\prime}} e^{-i q_{0} k \ell_{0}^{2}}\right] \\
& \times\left[C_{n, 1} C_{n^{\prime}, 1} M_{\left|n^{\prime}\right|-1,|n|-1}+C_{n, 4} C_{n^{\prime}, 4} M_{\left|n^{\prime}\right|+1,|n|+1}\right. \\
& \left.+\left(C_{n, 2} C_{n^{\prime}, 2}+C_{n, 3} C_{n^{\prime}, 3}\right) M_{\left|n^{\prime}\right|,|n|}\right],
\end{aligned}
$$

where

$$
M_{n^{\prime}, n}=\left(\frac{m !}{M !}\right)^{1 / 2} e^{-Q / 2} Q^{\left|n^{\prime}-n\right| / 2} L_{m}^{\left|n^{\prime}-n\right|}(Q),
$$




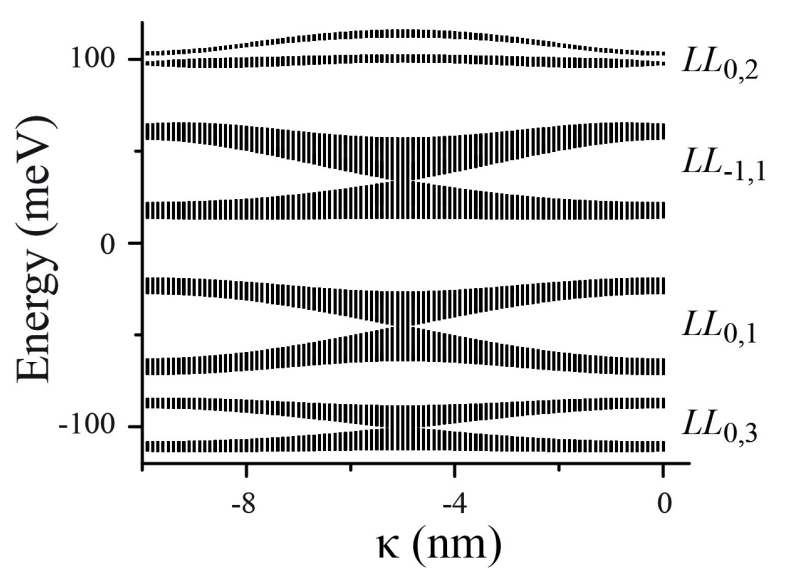

FIG. 5: Energy spectrum of the germanene monolayer as a function of the effective wave vector $\kappa$. The external electric field is zero. The amplitude of periodic potential is $40 \mathrm{meV}$ and its period is $20 \mathrm{~nm}$.

and $Q=q_{0}^{2} \ell_{0}^{2} / 2, m=\min \left(n^{\prime}, n\right), M=\max \left(n^{\prime}, n\right)$.

We study the regime of the strong magnetic field and consider the basis of four LLs, which correspond to $n=-1$ and $n=0$. Within these basis we construct the Hamiltonian matrix taking into account the matrix elements (11) and (12) of the periodic potential, and evaluate the corresponding energy spectrum as a function of parameter $\alpha=B a_{0}^{2} / \Phi_{0}$, where $\Phi_{0}=h /(2 e)$ is the magnetic flux quantum.

\section{NUMERICAL RESULTS AND DISCUSSION}

\section{A. The butterfly Energy Spectrum}

The energy spectra of buckled graphene-like materials in the regime of the strong magnetic field, i.e., weak periodic potential, is shown in Fig. 1 1 as a function of the parameter $\alpha$, which changes from 0 to 1 . We consider only four LLs with the label scheme shown in the figure, i.e., $L L_{-1,1}$ corresponds to the LL with index $n=-1$ (there is only one $L L$ with that index), while $L L_{0,1}, L L_{0,2}$, and $L L_{0,3}$ correspond to three LLs with index $n=0$. The results in Fig. 1 1 correspond to germanene, which has the largest SO interaction constant, $\lambda_{\mathrm{SO}}=43 \mathrm{meV}$. The results clearly illustrate the formation of the Hofstadter butterfly structures in each LL. For small $\alpha$, there is an overlap of the butterfly structures of the Landau levels $L L_{0,1}$ and $L L_{-1,1}$. Although the overlap is large, the coupling of $L L_{0,1}$ and $L L_{-1,1}$ states is weak. This is due to the fact that the overlap of the corresponding wave functions is small. For the zero external electric field, this overlap is exactly zero. For $\alpha>0.7$, i.e., in a weak magnetic field there is an overlap of the $L L_{0,1}$ and $L L_{0,2}$ Landau levels. In this case the overlap results in a relatively strong coupling of the corresponding states and modification of the energy spectrum.
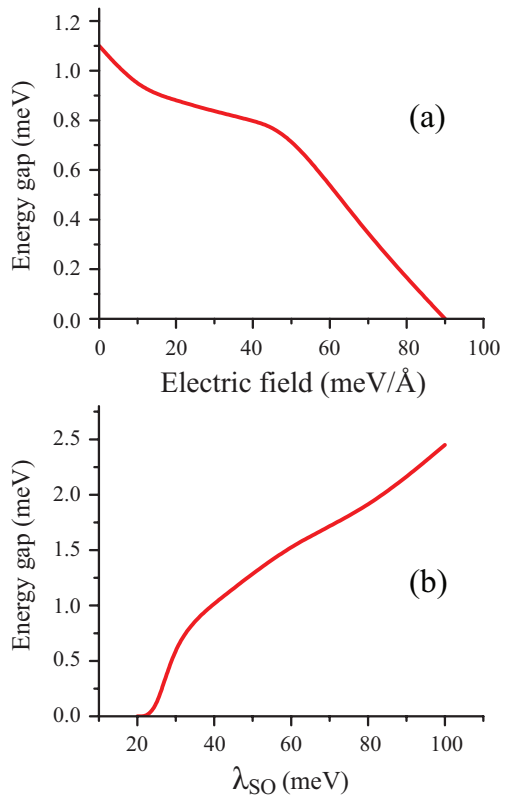

FIG. 6: Energy gap $\Delta$ between two bands in $L L_{0,2}$. (a) The period of the potential is $20 \mathrm{~nm}$ and its amplitude is $40 \mathrm{meV}$. The SO coupling is $\lambda_{\mathrm{SO}}=43 \mathrm{meV}$. (b) The electric field is 10 $\mathrm{mV} / \AA$. The period of the potential is $20 \mathrm{~nm}$ and its amplitude is $40 \mathrm{meV}$.

The results shown in Fig. 1 illustrate the typical structure of the energy spectra of buckled graphene-like materials. Variation of the external electric field, $E_{z}$, and the strength of the $\mathrm{SO}$ interaction, $\lambda_{\mathrm{SO}}$, change the energy spectra of the system. These changes can be described by studying the values of the energy gaps for rational $\alpha=p / q$, where $q-1$ gaps exist in each LL. For example, for $\alpha=1 / 3$ there are three bands and correspondingly two gaps in each LL, while for $\alpha=1 / 2$ there are two bands and one gap in each LL. In the case of overlapping LLs the gaps can also be closed. Below we study the properties of the energy spectra of buckled graphene-like materials for $\alpha=1 / 3$ and $\alpha=1 / 2$.

$$
\text { B. } \alpha=1 / 3
$$

For $\alpha=1 / 3$ there are three bands in each LL with the corresponding two gaps. In Fig. 2 the energy spectra for $\alpha=1 / 3$ are shown for four LLs and different amplitudes of the periodic potential, $V_{0}$. In each LL the three bands are clearly visible. With increasing $V_{0}$ the widths of the bands and correspondingly the band gaps increase. For large $V_{0}$ the coupling of the states of different LLs modifies the band structure of the LLs. We characterize this effect by studying the magnitudes of the two gaps marked in Fig. 2 by $\Delta_{1}$, which is the higher energy gap in the Landau level $L L_{-1,1}$, and $\Delta_{2}$, which is the lower energy gap in the Landau level $L L_{0,1}$.

The effect of the SO interaction strength on the ar- 
rangement of the LL bands is illustrated in Fig. 3, For small $\lambda_{\mathrm{SO}}(\lesssim 20 \mathrm{meV})$ the LLs $L L_{-1,1}$ and $L L_{0,1}$, which are broadened by the periodic potential, overlap that can close some gaps within these LLs. This corresponds to the case of silicene, for which $\lambda_{\mathrm{SO}}=3.9 \mathrm{meV}$. With increasing $\lambda_{\mathrm{SO}}$, the LLs $L L_{-1,1}$ and $L L_{0,1}$ become well separated. Compared to other LLs, the energies of the LLs $L L_{-1,1}$ and $L L_{0,1}$ have strong dependence on the SO coupling. This strong dependence results in the overlap of the LLs $L L_{0,1}$ and $L L_{0,3}$ for a large spin orbit coupling and large external electric field. The overlap of the LLs $L L_{0,1}$ and $L L_{0,3}$ is visible in Fig. 3 for $\lambda_{\mathrm{SO}}=100$ $\mathrm{meV}$ and the electric field $E_{z}=100 \mathrm{mV} / \AA$, while for $E_{z}=0$ there is no overlap of the LLs even for large $\lambda_{\mathrm{SO}}$. In Fig. 4. the dependence of the energy gaps $\Delta_{1}$ and $\Delta_{2}$, which are defined in Fig. 2, on the SO coupling, $\lambda_{\mathrm{SO}}$. For zero electric field [Fig. 2(a)] the gaps $\Delta_{1}$ and $\Delta_{2}$ are exactly the same. In this case the dependence of $\Delta_{1}$ on the SO interaction strength is relatively weak. The gap $\Delta_{1}$ changes by only $\approx 15 \%$ when the coupling $\lambda_{\mathrm{SO}}$ increases from $3 \mathrm{meV}$ (silicene) to $100 \mathrm{meV}$.

The dependence of the gaps $\Delta_{1}$ and $\Delta_{2}$ on $\lambda_{\mathrm{SO}}$ becomes more pronounced for large electric fields. The gaps $\Delta_{1}$ and $\Delta_{2}$ in this case are not equal. The gap $\Delta_{2}$ still has a very weak dependence on $\lambda_{\mathrm{SO}}$. This gap corresponds to the high energy gap for $L L_{-1,1}$. At the same time, the gap $\Delta_{1}$ has a very strong dependence on $\lambda_{\mathrm{SO}}$ and with increasing $\lambda_{\text {SO }}$ it is strongly suppressed [Fig. [4(b)]. The gap $\Delta_{1}$ changes from $2 \mathrm{meV}$ for small $\lambda_{\mathrm{SO}} \approx 3 \mathrm{meV}$ to $0.8 \mathrm{meV}$ for large $\lambda_{\mathrm{SO}} \approx 100 \mathrm{meV}$. This suppression is due to the fact that with increasing $\lambda_{\mathrm{SO}}$ the separation between the LLs $L L_{0,1}$ and $L L_{0,3}$ decreases (see Fig. 3), which results in stronger coupling of the states of these LLs, and correspondingly a strong change in the value of the gap $\Delta_{1}$. The gap, $\Delta_{1}$, has also a nonmonotonic dependence on $\lambda_{\mathrm{SO}}$, which is more pronounced for larger amplitudes of the periodic potential, $V_{0}$. As an example, for $V_{0}=20 \mathrm{meV}$ (see Fig. प(c)), the gap $\Delta_{1}$ first increases from small value of $\approx 2.5 \mathrm{meV}$ for small $\lambda_{\mathrm{SO}}$, then reaches its maximum of $\approx 6.5 \mathrm{meV}$ for $\lambda_{\mathrm{SO}} \approx 20$ $\mathrm{meV}$, and finally decreases for large $\lambda_{\mathrm{SO}}$.

$$
\text { C. } \alpha=1 / 2
$$

For $\alpha=1 / 2$ there are two bands in each LL. Specific to this case is the fact that the gap between these two bands in each LL is zero. A gap can however be opened by the Coulomb interaction between electrons [44, 45]. In the case of buckled graphene-like materials there are other parameters, the SO coupling and the external electric field, that can modify the band structure of the LLs. To characterize the band structure of the LLs, we introduce an "effective 2D wave vector", $\left(\kappa_{x}, \kappa_{y}\right)$, which reflects the periodicity of the wave functions in the reciprocal space. This periodicity follows from the expressions of the matrix elements of the periodic potential [Eqs. (11)-(12)]. The effective wave vector $\left(\kappa_{x}, \kappa_{y}\right)$ has the units of length and are defined within an effective Brillouin zone. In Fig. 5 the typical energy spectrum of the germanene layer for $\alpha=1 / 2$ is shown as a function of $\kappa_{x}$ and different values of $\kappa_{y}$. The spectrum clearly shows two bands in each LL. The gaps between the bands are zero for the LLs $L L_{-1,1}, L L_{0,1}$, and $L L_{0,3}$, while there is a finite gap $\Delta$ for $L L_{0,2}$. In Fig. [6 the gap $\Delta$ is shown as a function of the electric field and the SO coupling. With increasing electric field the gap decreases and finally disappears for large fields. As a function of the SO interaction the gap increases with $\lambda_{\mathrm{SO}}$, which illustrates the fact that the gap is due to the strong SO coupling in the system. For small SO coupling, $\lambda \lesssim 20 \mathrm{meV}$, the gap is zero and it monotonically increases with $\lambda_{\mathrm{SO}}$ reaching the value of $2.5 \mathrm{meV}$ for $\lambda_{\mathrm{SO}}=100 \mathrm{meV}$. Therefore, opening of the gap for $\lambda=1 / 2$ can be observed only in the germanene monolayer which has a large SO coupling, $\lambda \approx 40 \mathrm{meV}$, while in the silicene monolayer with small SO coupling, $\lambda_{\mathrm{SO}} \approx 3.4 \mathrm{meV}$, the gap is zero.

\section{CONCLUSION}

The graphene-like materials, e.g., silicene and germanene, have strong SO interaction and the buckled structure that results in these materials being sensitive to a perpendicular electric field. In this case the main parameters, which determines the unique properties of these materials, are the SO coupling, $\lambda_{\mathrm{SO}}$, and the external perpendicular electric field, $E_{z}$. The properties of buckled graphene-like materials in a magnetic field and the periodic potential also depend on the values of these parameters. That dependence can be described in terms of the dependence of the band structure of the LLs for rational values of $\alpha$. One of the characteristics of the band structure is the gap between the intra-Landau level bands. For $\alpha=1 / 3$, some gaps show strong dependence on both the electric field and the SO coupling. For a large electric field and large amplitude of the periodic potential, the dependence of the gap on the SO coupling is highly nonmonotonic. For $\alpha=1 / 2$, without the SO coupling and for all values of the electric field, all gaps are closed. For a large SO interaction, $\lambda_{\mathrm{SO}}>20 \mathrm{meV}$, which is realized in germanene, the gap in one of the LLs opens. The magnitude of the gap increases with $\lambda_{\mathrm{SO}}$ and decreases with the electric field. Experimental confirmation of these predictions will provide a rare peek into the electronic properties of these unique materials with emerging properties. Finally, the influence of the electron-electron interaction on the butterfly gap structure is an important direction for exploration in these and other similar 47] Dirac materials, that has already seen some progress, theoretically and experimentally, in graphene [44-46]. 


\section{ACKNOWLEDGMENTS}

The work has been supported by the Canada Research

Chairs Program of the Government of Canada.

[ $\ddagger$ Electronic address: Tapash.Chakraborty@umanitoba.ca

[2] U. Rössler and M. Shurke, in Advances in Solid State Physics, edited by B. Kramer (Springer, Berlin 2000), Vol. 40, pp. 35-50.

[3] P.G. Harper, Proc. Phys. Soc. London 68, 874 (1955).

[4] D. Langbein, Phys. Rev. 180, 633 (1969).

[5] D.R. Hofstadter, Phys. Rev. B 14, 2239 (1976).

[6] M.C. Geisler, J. H. Smet, V. Umansky, K. von Klitzing, B. Naundorf, R. Ketzmerick, and H. Schweizer, Phys. Rev. Lett. 92, 256801 (2004); C. Albrecht, J. H. Smet, K. von Klitzing, D. Weiss, V. Umansky, and H. Schweizer, Phys. Rev. Lett. 86, 147 (2001); U. Kuhl and H. J. Stöckmann, Phys. Rev. Lett. 80, 3232 (1998); M. Aidelsburger, M. Atala, M. Lohse, J. T. Barreiro, B. Paredes, and I. Bloch, Phys. Rev. Lett. 111, 185301 (2013); H. Miyake, G. A. Siviloglou, C. J. Kennedy, W. C. Burton, and W. Ketterle, Phys. Rev. Lett. 111, 185302 (2013).

[7] J.-W. Rhim and K. Park, Phys. Rev. B 86, 235411 (2012).

[8] N. Nemec and G. Cuniberti, Phys. Rev. B 75, 201404 (2007).

[9] R. Bistritzer and A. H. MacDonald, Phys. Rev. B 84, 035440 (2011).

[10] C.R. Dean, L. Wang, P. Maher, C. Forsythe, F. Ghahari, Y. Gao, J. Katoch, M. Ishigami, P. Moon, M. Koshino, T. Taniguchi, K.Watanabe, K.L. Shepard, J.Hone, and P. Kim, Nature 497, 598 (2013).

[11] B. Hunt, J.D. Sanchez-Yamagishi, A.F. Young, M. Yankowitz, B.J. LeRoy, K. Watanabe, T. Taniguchi, P. Moon, M. Koshino, P. Jarillo-Herrero, and R.C. Ashoori, Science 340, 1427 (2013).

[12] L.A. Ponomarenko, R.V. Gorbachev, G.L. Yu, D.C. Elias, R. Jalil, A.A. Patel, A. Mishchenko, A.S. Mayorov, C.R. Woods, J.R. Wallbank, M. Mucha-Kruczynski, B.A. Piot, M. Potemski, I.V. Grigorieva, K.S. Novoselov, F. Guinea, V.I. Falko and A.K. Geim, Nature 497, 594 (2013).

[13] T. Chakraborty and V. M. Apalkov, IET Circuits Devices Syst. 9, 19 (2015) [DOI: 10.1049/iet-cds.2014.0275].

[14] H. Aoki and M.S. Dresselhaus (Eds.), Physics of Graphene (Springer, New York 2014) [DOI: 10.1007/9783-319-02633-6].

[15] D.S.L. Abergel, V. Apalkov, J. Berashevich, K. Ziegler, and T. Chakraborty, Adv. Phys. 59, 261 (2010).

[16] T. Chakraborty and V. Apalkov, in [14], Chapter 8 [DOI: 10.1007/978-3-319-02633-6-8].

[17] R. Decker, Y. Wang, V. W. Brar, W. Regan, H.-Z. Tsai, Q. Wu, W. Gannett, A. Zettl, and M. F. Crommie, Nano Lett. 11, 2291 (2011).

[18] J. Xue, J. Sanchez-Yamagishi, D. Bulmash, P. Jacquod, A. Deshpande, K. Watanabe, T. Taniguchi, P. JarilloHerrero, and B. J. LeRoy, Nat. Mater. 10, 282 (2011).

[19] A. Kara, H. Enriquez, A.P. Seitsonen, L.C. Lew Yan Voon, S. Vizzini, B. Aufray, and H. Oughaddou, Surf. Sci. Rep. 67, 1 (2012).
[20] Q. Tang and Z. Zhou, Progr. Mater. Sci. 58, 1244 (2013).

[21] H. Rostami, A. Moghaddam, and R. Asgari, Phys. Rev. B 88, 085440 (2013).

[22] A. Kormnyos, V. Zlyomi, N. Drummond, P. Rakyta, G. Burkard, and V. Falko, Phys. Rev. B 88, 045416 (2013).

[23] K. Takeda and K. Shiraishi, Phys. Rev. B 50, 14916 (1994).

[24] C.-C. Liu, W. Feng, and Y. Yao, Phys. Rev. Lett. 107, 076802 (2011).

[25] C.-C. Liu, H. Jiang, and Y. Yao, Phys. Rev. B 84, 195430 (2011).

[26] Y.Wang, J. Zheng, Z. Ni, R. Fei, Q. Liu, R. Quhe, C. Xu, J. Zhou, Z. Gao, and J. Lu, Nano 07, 1250037 (2012).

[27] F.-b. Zheng and C.-w. Zhang, Nanoscale Res. Lett. 7, 422 (2012).

[28] B. Lalmi, H. Oughaddou, H. Enriquez, A. Kara, S. Vizzini, B. Ealet, and B. Aufray, Appl. Phys. Lett. 97, 223109 (2010).

[29] P. De Padova, C. Quaresima, B. Olivieri, P. Perfetti, and G. Le Lay, Appl. Phys. Lett. 98, 081909 (2011).

[30] P. Vogt, P. De Padova, C. Quaresima, J. Avila, E. Frantzeskakis, M. C. Asensio, A. Resta, B. Ealet, and G. Le Lay, Phys. Rev. Lett. 108, 155501 (2012).

[31] L. Chen, C.-C. Liu, B. Feng, X. He, P. Cheng, Z. Ding, S. Meng, Y. Yao, and K. Wu, Phys. Rev. Lett. 109, 056804 (2012).

[32] L. Chun-Liang, A. Ryuichi, K. Kazuaki, T. Noriyuki, M. Emi, K. Yousoo, T. Noriaki, and K. Maki, Appl. Phys. Expr. 5, 045802 (2012).

[33] B. Feng, Z. Ding, S. Meng, Y. Yao, X. He, P. Cheng, L. Chen, and K. Wu, Nano Lett. 12, 3507 (2012).

[34] A. Fleurence, R. Friedlein, T. Ozaki, H. Kawai, Y.Wang, and Y. Yamada-Takamura, Phys. Rev. Lett. 108, 245501 (2012).

[35] M. E. Dvila, L. Xian, S. Cahangirov, A. Rubio, and G. L. Lay, New J. Phys. 16, 095002 (2014).

[36] L. Li, S.-z. Lu, J. Pan, Z. Qin, Y.-q. Wang, Y. Wang, G.-y. Cao, S. Du, and H.-J. Gao, Adv. Mat. 26, 4820 (2014).

[37] L. Meng, Y. Wang, L. Zhang, S. Du, R. Wu, L. Li, Y. Zhang, G. Li, H. Zhou, W. A. Hofer, et al., Nano Lett. 13, 685 (2013).

[38] C.-C. Liu, H. Jiang, and Y. Yao, Phys. Rev. B 84, 195430 (2011).

[39] M. Gmitra, S. Konschuh, C. Ertler, C. Ambrosch-Draxl, and J. Fabian, Phys. Rev. B 80, 235431 (2009).

[40] E. Motohiko, New J. Phys. 14, 033003 (2012).

[41] V.M. Apalkov and T. Chakraborty, Phys. Rev. B 90, 245108 (2014).

[42] M. Califano, T. Chakraborty and P. Pietiläinen, Phys. Rev. Lett. 94, 246801 (2005); D.S.L. Abergel and T. Chakraborty, Phys. Rev. Lett. 102, 056807 (2009); V.M. Apalkov and T. Chakraborty, Phys. Rev. Lett. 97, 126801 (2006); Phys. Rev. Lett. 105, 036801 (2010); Phys. Rev. Lett. 107, 186803 (2011); V.M. Apalkov, T 
Chakraborty, P Pietiiäinen, K Niemeä, Phys. Rev. Lett. 87, 1311 (2001); T. Chakraborty and P. Pietiäinen, Phys. Rev. Lett. 76, 4018 (1996).

[43] M. Ezawa, J. Phys. Soc. Jpn. 81, 064705 (2012).

[44] V.M. Apalkov and T. Chakraborty, Phys. Rev. Lett. 112, 176401 (2014).

[45] A. Ghazaryan and T. Chakraborty, Phys. Rev. B 91, 125131 (2015); A. Ghazaryan, T. Chakraborty, and P. Pietiläinen, J. Phys.: Condens. Matter $\mathbf{x x}$, xxxxxx (2015).
[46] G. L. Yu, R. V. Gorbachev, J. S. Tu, A. V. Kretinin, Y. Cao, R. Jalil, F.Withers, L. A. Ponomarenko, B. A. Piot, M. Potemski, D. C. Elias, X. Chen,K.Watanabe, T. Taniguchi, I.V.Grigorieva, K. S. Novoselov, V. I. Falko, A. K. Geim, and A. Mishchenko, Nat. Phys. 10, 525 (2014).

[47] Yen-Hung Ho, Wu-Pei Su and Ming-Fa Lin, RSC Adv., 5, 20858 (2015). 